\title{
Scientists closing in on true identity of the 'great imitator'
}

Imagine a disease with no cure, no known cause and an elusive diagnosis. Dubbed the 'great imitator' because it mimics so many other diseases, systemic lupus erythematosus (SLE) has remained an enigma-until now. With new genetic technologies and the complete sequence of the human genome, scientists are poised to take a quantum leap in unraveling its mysteries.

SLE is an autoimmune disease where the body attacks its own cells and tissues, causing inflammation in various parts of the body, including the skin, kidneys, heart and nervous system. It affects 1.4 million people in the US alone. In its worst form, lupus can be fatal: 15\% of patients die from kidney disease, cardiovascular disease or infections.

The discovery of potential biomarkers will soon allow doctors to predict the severity of the disease, says Timothy W. Behrens, professor of rheumatology and autoimmune diseases at the University of Minnesota. "We are very close to the identification of several of the genes that increase a person's chances for developing lupus," Behrens says. Behrens and others presented their research at a meeting in September in Washington, D.C.

Using gene expression microarrays, Behrens and his colleagues have identified a 'signature' of genes activated by interferon in patients with lupus. Although the precise biochemistry is unknown, evidence suggests that the disease is somehow activating the interferon pathway, says Behrens. The researchers analyzed thousands of genes in the peripheral blood cells of 48 lupus patients and 42 healthy controls.

Nearly $80 \%$ of the SLE patients have higher expression of 14 genes, referred to collectively as the interferon gene expression signature, the researchers found (Proc. Natl. Acad. Sci. USA $100,2610-2615 ; 2003)$. The signature seems to be a marker for patients with the more severe type of lupus, which can involve the brain, kidneys and blood cells, Behrens says.

"We believe that there is potential for the development of new diagnostic tests based on the signature," says Behrens. Many patients now go up to four years before a definite diagnosis is made. "In the near future," Behrens adds, "these types of data - the genes a person carries and the pattern of gene expression in their blood cells - will be used by doctors to individualize therapy in a way that has never been possible."

The results have also spurred interest in initiating clinical trials with drugs that block the interferon pathway. Several trials are under way to explore manipulation of chemical pathways

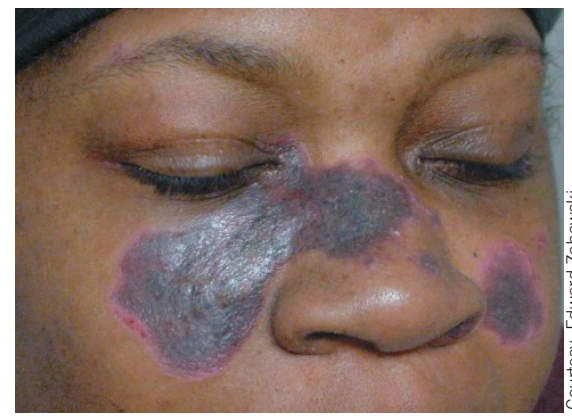

Skin deep: The characteristic butterfly rash in lupus can be indicative of a severe disease affecting internal organs.

in lupus patients. A phase 1 clinical trial sponsored by the US National Institutes of Health is investigating the safety and effects of the monoclonal antibody MRA, which blocks the action of interleukin-6, thought to lead to organ damage in SLE patients.

The US National Institute of Allergy and Infectious Diseases is conducting a phase 1 study that explores the possibility of B-cell depletion as a potential treatment for lupus.

\section{Japanese scientists plan MMR alternative}

The Japanese government has approved a fiveyear grant, with $¥ 56$ million slated for the first year, to create a single recombinant vaccine for four common childhood diseases. Researchers at Osaka University and Toyama Medical and Pharmaceutical University plan to insert genes encoding the antigens for measles, mumps and rubella into the varicella (chickenpox) virus. There are currently no recombinant vaccines against multiple diseases licensed for human use.

Improving vaccine coverage, particularly for measles, is a priority for Japanese health officials, says Keiko Taya, chief of the immunization program at Japan's Infectious Disease Surveillance Center. The government is currently assessing its vaccine policiesstatistics indicate that measles coverage in 2001 was only $50 \%$ for one-year-olds, and that adult cases are on the rise.

According to project leader Koichi Yamanishi, the researchers decided to use varicella as the vector because it has a relatively large amount of dispensable DNA, which makes it a good candidate for accepting foreign genes. The team-which includes Michiaki Takahashi, who developed the Oka varicella vaccine now used worldwide- has already successfully expressed hepatitis B and
Because they produce autoantibodies, B cells have an essential role in lupus pathogenesis. Rituxan, an antibody to CD20, can block production of B cells in patients with nonHodgkin lymphoma. Researchers hope that by interrupting autoantibody production by the $\mathrm{B}$ lymphocyte, Rituxan might be an effective therapy for lupus as well.

Lupus is more common in women of Hispanic, Asian and Native American descent. Children and adolescents represent $15 \%$ of SLE patients. As more afflicted children survive into adulthood, atherosclerotic cardiovascular disease has emerged as a major concern.

Laura Schanberg, associate professor at Duke University Medical Center, is planning to test whether the statin Lipitor can reduce cholesterol levels in children with lupus. "This is the first ever drug intervention trial in pediatric SLE," says Schanberg. The researchers have started enrolling patients into the trial at a few sites, with the rest of the sites expected to start enrolling later this year.

Amy K. Erickson, Washington, D.C.

\section{HIV antigens in varicella.}

Another advantage of varicella is that compared with other vaccines, such as measles, its side effects are relatively mild. "The varicella backbone is very safe," says Anne Gershon, director of the pediatric infectious disease division at Columbia University. But Gershon cautions that the safety and efficacy testing process could take a long time- the researchers aim to have a vaccine ready for clinical trials within five years. Gershon also notes that current vaccines are safe and effective.

Yamanishi says Japan's problem may not be related to the effectiveness of the vaccines, but to the time it takes to receive them. Japanese children now receive four separate vaccinations for measles, mumps, rubella and varicella, a process prone to delays. Japan discontinued use of the MMR vaccine in 1993 over concerns that the Urabe mumps strain used in Japanese MMR vaccines was associated with adverse side effects such as meningitis. A new MMR vaccine isn't likely to be available for several more years. "If [a child] catches even a common cold during [that] year, the next injection gets delayed," says Yamanishi. "One injection could be much more convenient."

I-han Chou, Tokyo 